\title{
Research on Herbal Fumigation Treatment of Psoriasis
}

\author{
Yanfeng Lou \\ Nanjing General Hospital of Nanjing Military Region, Nanjing Jiangsu, 210002, China
}

Keywords: Psoriasis, Herbal fumigation, Nursing.

\begin{abstract}
Objective: To investigate the nursing method and effect of herbal fumigation treatment of psoriasis. Method: choose 100 cases of psoriasis patients in our hospital from January 2012 to December 2015, as the object of the study, take computer random selection method to divide these 100 patients into control group and observation group, with 50 patients in each group. All patients receive herbal fumigation treatment, and during treatment, control group receives routine nursing intervention, while observation group receives targeted nursing intervention. Results: The clinical total effective rate of observation group is $96 \%$, significantly higher $(\mathrm{P}<0.05)$ than $80 \%$ of control group. Adverse reactions occurrence rate of observation group is $14 \%$, significantly lower $(\mathrm{P}<0.05)$ than $32 \%$ of control group. Recurrence rate of of observation group is $26 \%$, significantly lower (P $<0.05$ ) than $46 \%$ of control group. Compared with pre-intervention, after intervention, anxiety score and sleep quality index in both groups are significantly lower $(\mathrm{P}<0.05)$, but anxiety score and sleep quality index in observation group after the intervention is significantly lower $(\mathrm{P}<0.05)$ compared with control group. Conclusion: Herbal fumigation treatment of psoriasis, in line with the targeted nursing intervention, can effectively ensure the therapeutic effect, improve skin damage condition and reduce adverse reactions and recurrence, ease unhealthy mood, and improve sleep quality.
\end{abstract}

\section{Introduction}

Psoriasis is a common chronic inflammatory skin disease, often called "psora", persistent, and easy to relapse, with clinical manifestations of erythema, scaling, often occurring on the scalp and limbs, harmful to the patient' s physical and mental health, so clinically it is necessary to take active and effective treatment on psoriasis ${ }^{[1]}$. Herbal fumigation is the primary means of treatment of psoriasis, mainly through the use of Chinese herbal to fumigate and rinse the affected area, so as to achieve the purpose of treatment, and also with the appropriate nursing measures needed in herbal fumigation process. To investigate the nursing method and effect of herbal fumigation treatment of psoriasis, the study chooses 100 cases of psoriasis patients in our hospital from January 2012 to December 2015, as the object of the study, all receiving herbal fumigation treatment, and routine nursing and targeted nursing respectively implemented during treatment. Now we have finished the analysis on research data and report the study as follows.

\section{Data and Method}

\section{Basic data.}

Choose 100 cases of psoriasis patients in our hospital from January 2012 to December 2015, as the object of the study, all diagnosed with psoriasis, with TCM syndrome differentiation of dryness type and blood heat type, and exclude patients with herbal fumigation contraindication, cardiac dysfunction, taking steroids or other topical medications in nearly two weeks, or lacking clinical data. Before the study, all patients are voluntary to participate in the study, and have the right to know the method and purpose of the study. The research is approved by Hospital Ethics Committee.

Take computer random selection method to divide these 100 patients into control group and observation group, with 50 patients in each group. In control group, there are 27 males and 23 females; the youngest is 21 years old, the oldest is 69 years old, and mean age is ( $45.17 \pm 15.62)$; sick time is as short as 6 months or as long as 5 years, and the average is ( $2.93 \pm 1.09)$ years. In observation group, there are 28 males and 22 females; the youngest is 20 years old, the oldest is 70 years old, and 
mean age is (45.29 \pm 15.86$)$ years; sick time is as short as 5 months, or as long as 6 years, and the average is ( $3.07 \pm 1.14$ ) years.

Compare the baseline information of patients in two groups including gender, mean age, average sick time, use $\mathrm{x}^{2}$ test or $\mathrm{t}$ test to get $\mathrm{P}>0.05$, which indicates the difference is not statistically significant, equilibrium between the basic information of two groups maintains good and they can be compared.

\section{Method.}

1) Treatment method

All patients receive herbal fumigation treatment. The prescription contains, $30 \mathrm{~g}$ of cortex dictamni, $30 \mathrm{~g}$ of cortex lycii radicis, $30 \mathrm{~g}$ of cacumen biotae, $30 \mathrm{~g}$ of golden cypress, $30 \mathrm{~g}$ of pepper, and $30 \mathrm{~g}$ of folium artemisiae argyi. Add water to boil out until the concoction remains $4 \mathrm{~L}$, add 40L of warm water at 1:10 for dilution, put diluted liquid into wooden tub, control the temperature at about $37^{\circ} \mathrm{C}$, and let the patient sit in the tub, with the water level controlled at the patient chest, drying after fumigation for 20 minutes. Take once a day.

\section{2) Nursing method}

During treatment, control group receives routine nursing intervention, as follows. Inform patients before fumigation with fumigation method and caveats, and patients need to empty their bladder; during fumigation process monitor and control temperature, humidity should be maintained at suitable state, and closely observe the skin of the patient, if the patient has abnormalities, should end fumigation immediately; after fumigation, check the patient' s skin, to see if there occurs new skin damage and help the patient put on clothing, and pay attention to keeping warm.

Observation group receives targeted nursing intervention on the basis of routine nursing intervention, and specific measures are as follows: (1) health guidance: evaluate disease awareness of patients, based on the awareness degree, conduct targeted health guidance, and inform patients of related matters in daily life to be noticed, to avoid the potential risk factors in life, and inform patients to pay attention to exercise training to improve local circulation. (2) diet nursing: according to the specific conditions of the patient, conduct targeted dietary guidance for the patient. The patient should keep light diet, increase intake of fiber, vitamins, protein in daily diet, give up tobacco and alcohol, spicy, cold and other irritable food. (3)emotional nursing: make active communication and exchange with the patient, understand the psychological dynamics of the patient, appropriately appease the patient, to guide him to rebuild a positive self-awareness, improve his confidence on treatment, and effectively alleviate his stress, anxiety and other emotions. (4) acupoint massage: take specific acupoints, such as Sanyinjiao, Quchi, Xuehai, Shenmen, Geshu, and press and rub corresponding acupoints.

\section{Observation index.}

After treatment, compare clinical total efficiency, occurrence rate of adverse reactions, anxiety score and sleep quality index after intervention of two groups, conduct three-month follow-up observation, and conclude the recurrence result.

\section{Evaluation criteria ${ }^{[2]}$.}

Total score of skin damage score (PASI) is 72 points, including the skin damage of head, arms, legs, and torso. The higher the score is, the severer skin damage is.

Curative effect criteria: recovery, namely, PASI score reduction reaches 90\%; markedly effective, namely, PASI score reduction reaches $60 \%$, but less than $90 \%$; effective, namely, PASI score reduction reaches $25 \%$, but less than $60 \%$; ineffective, namely, PASI score reduction is less than $25 \%$. The clinical total effective rate $=$ (recovery cases + markedly effective cases + effective cases $)$ / total cases $\times 100 \%$.

Total score of anxiety score (SAS) is 100 points, with critical value of 50 points. The higher the score is, the severer anxiety is.

Total score of sleep quality index (PSQI) is 0 to 21 points. Higher score indicates worse sleep quality. 


\section{Statistic method.}

Use SPSS19.0 software to process data, count data (gender, clinical total effective rate, occurrence rate of adverse reactions, the recurrence rate) and measurement data (mean age, average sick time, anxiety score, sleep quality index), conduct $x^{2}$ test, t-test, expressed as [n (\%)], $(\bar{x} \pm s)$. When $\mathrm{P}<0.05$, the difference is statistically significant.

\section{Results}

\section{Comparison of clinical total effective rate of two groups.}

In observation group, there are 13 recovery cases, 21 markedly effective cases, 14 effective cases, 2 ineffective cases; while in control group: there are 8 recovery cases, 17 markedly effective cases, 15 effective cases, 10 ineffective cases. Clinical total effective rate of observation group is 96\%, significantly higher $(\mathrm{P}<0.05)$ than $80 \%$ of control group.

Comparison of occurrence rate of adverse reactions of two groups.

In observation group, there are 7 cases of adverse reactions ( 3 cases of pruritus, 2 cases of burning, 2 cases of drying), while control group has 16 cases of adverse reactions (5 cases of pruritus, 3 cases of erythema, 4 cases of burning, 4 cases of drying). Occurrence rate of adverse reactions of observation group is $14 \%$, significantly lower $(\mathrm{P}<0.05)$ than $32 \%$ of control group.

\section{Comparison of recurrence rate of two groups.}

After follow-up visit, it is found in the observation group, there are 13 cases of recurrence, while control group has 23 cases. Recurrence rate of observation group is $26 \%$, significantly lower $(\mathrm{P}<0.05)$ than $46 \%$ of control group.

Comparison of anxiety score and sleep quality index after intervention of two groups.

Before intervention, in control group, anxiety score and sleep quality index are respectively (19.72 \pm 3.83$)$ points, and $(16.18 \pm 2.53)$ points, while in observation group, anxiety score and sleep quality index are respectively (19.74 \pm 3.78$)$ points, and (16.27 \pm 2.59 ) points; after intervention, in control group, anxiety score and sleep quality index are respectively (10.56 \pm 2.49$)$ points, and (11.69 $\pm 2.09)$ points, while in observation group, anxiety score and sleep quality index are respectively $(6.93 \pm 2.86)$ points, and $(8.23 \pm 1.74)$ points. Compared with pre-intervention, after intervention, anxiety score and sleep quality index in both groups are significantly lower $(\mathrm{P}<0.05)$, but anxiety score and sleep quality index in observation group after the intervention is significantly lower ( $\mathrm{P}$ $<0.05)$ compared with control group.

\section{Discussion}

Psoriasis is a relatively common chronic skin inflammatory lesion, with clinical manifestations of scaling and erythema, mainly occurring on the scalp and limbs, often accompanied by skin lesions, not only affecting the physical health of patients, but also interfering their mental health. Because of lingering course of psoriasis, and delayed healing of the disease, if patients fail to receive timely treatment, it is likely to develop into a systemic and systemic damage. If treatment on patients is not good, and it is likely to recur, so clinically we should take active and effective treatment on psoriasis.

Herbal fumigation is a commonly used method of Chinese medicine treatment, mainly referred to the way of fumigation, by which, the drug efficacy directly contacts with lesions with the aid of heat, able to quickly onset, and wash the affected area ${ }^{[3]}$. The herbal fumigation in this study is mainly composed of cortex dictamni, cortex lycii radicis, cacumen biotae, golden cypress, pepper, and folium artemisiae argyi, wherein, cortex dictamni, and golden cypress can clear heat and expel dampness; cortex lycii radicis and cacumen biotae can clear away heat and toxic materials, cool blood and disperse blood stasis, pepper and folium artemisiae argyi can stop itching and sterilize. Psoriasis belongs to blood heat syndrome or blood dryness syndrome, and treatment principles of Chinese medicine are cooling blood and dispersing blood stasis, clearing heat and dampness, therefore, the application of above herbs for fumigation treatment can be effective in treating psoriasis ${ }^{[4-5]}$. 
In the study, observation group also takes targeted nursing interventions, including dietary intervention, health guidance, emotional nursing, acupoint massage, wherein, dietary intervention can effectively avoid risk factors in terms of diet; health guidance can prompt patients to positively avoid risk factors in daily life and reduce recurrence; emotional nursing can effectively relieve unhealthy emotions of patients; and acupoint massage can effectively stimulate the local blood supply. The results of this study show that in observation group, taking targeted nursing, clinical total effective rate is $96 \%$, occurrence rate of adverse reactions is $14 \%$, and the recurrence rate is $26 \%$, significantly better $(\mathrm{P}<0.05)$ compared with control group taking routine nursing, indicating that herbal fumigation treatment plus targeted nursing can effectively promote the repair of skin lesions, reduce adverse reactions and recurrence; after intervention, observation group has significantly lower $(\mathrm{P}<0.05)$ anxiety score and sleep quality index compared with control group, indicating that targeted nursing can effectively improve sleep quality and psychological state of patients.

In summary, herbal fumigation method for the treatment of psoriasis in line with targeted nursing intervention can effectively ensure the therapeutic effect, improve skin damage condition, reduce adverse reactions and recurrence, alleviate unhealthy emotions, and improve sleep quality.

\section{References}

[1] S J Ersser. Providing lifestyle behaviour change support for patients with psoriasis: an assessment of the existing training competencies across medical and nursing health professionals, British Journal of Dermatology,2014,171(3):450-451.

[2] Huang Cuiwei. Herbal Fumigation and NB-UVB Treat 28 Cases of Plaque Psoriasis, Chinese Medicine Modern Distance Education, 2015,13 (10): 41-43.

[3] Sun Yunli. Herbal Fumigation Adjuvant Treatment of Psoriatic Arthritis Rash, Chinese Folk Medicine, 2015,23 (8): 26-27.

[4] Ersser S J, Cowdell F C, Nicholls P G, et al. A pilot randomized controlled trial to examine the feasibility and efficacy of an educational nursing intervention to improve self-management practices in patients with mild-moderate psoriasis, Journal of the European Academy of Dermatology and Venereology: JEADV,2012,26(6):738-745.

[5] Chen Hua. Clinical Efficacy Observation of Chinese Traditional Medical Nursing of Psoriasis, Chinese Community Doctors (medical profession), 2013,15 (8): 215. 